\title{
Induction of the marsupial sperm acrosome reaction in vitro by treatment with diacylglycerols
}

\author{
Y. Sistina* ${ }^{*}$ M. Lin, K. E. Mate† and J. C. Rodger \\ Department of Biological Sciences, University of Newcastle, NSW 2308, Australia
}

\begin{abstract}
The diacylglycerol $\mathrm{DiC}_{8}$ (1,2-dioctanoyl-sn-glycerol; $100 \mu \mathrm{mol} \mathrm{l}^{-1}$ ) was found to induce acrosomal loss in $70 \%$ of wallaby spermatozoa and $40 \%$ of possum spermatozoa after incubation for $120 \mathrm{~min}$. If $10 \mu \mathrm{mol}$ calcium ionophore $\mathrm{l}^{-1}$ was present, the time required to reach these end points was reduced to 30 and $60 \mathrm{~min}$, respectively. The diacylglycerol OAG (1oleoyl-2-acetyl-sn-glycerol; $100 \mu \mathrm{mol}^{-1}$ ) produced some acrosomal loss, particularly when $10 \mu \mathrm{mol}$ calcium ionophore $\mathrm{I}^{-1}$ was present, but when the ionophore was absent, results were equivocal. The other diacylglycerol tested DOG (1,2-dioleoyl-sn-glycerol; $100 \mu \mathrm{mol}$ $1^{-1}$ ) did not induce significant acrosomal loss in possum or wallaby spermatozoa. The concentration of $\mathrm{DiC}_{8}$ required to induce acrosomal loss in marsupial spermatozoa (50-100 $\mu \mathrm{mol}$ $\mathrm{I}^{-1}$ ) was relatively high compared with that for placental mammals, suggesting a less specific mode of action than enzyme activation. Of the diacylglycerols tested alone, only $\mathrm{DiC}_{8}$ led to significant loss of motility. In wallabies, this was detectable after incubation for $60 \mathrm{~min}$ and in possums after incubation for $120 \mathrm{~min}$. The ultrastructure of the $\mathrm{DiC}_{8}$-induced acrosomal loss was essentially identical to the acrosome reaction described for a broad range of placental mammals. A membrane vesicle shroud covered the acrosomal surface of the sperm head. The vesicles appeared to be formed by multiple point fusions between the plasma membrane and the outer acrosomal membrane. The mechanism of $\mathrm{DiC}_{8}$-induced acrosomal loss remains to be established. However, fusion of plasma membrane with outer acrosomal membrane was evident after incubation for $5 \mathrm{~min}$ in the diacylglycerol, long before a significant decline in motility was detected.
\end{abstract}

\section{Introduction}

During fertilization the spermatozoa of placental mammals undergo the acrosome reaction, an exocytotic event required for penetration of the oocyte vestments and fusion with the oocyte plasma membrane (Barros et al., 1967; Yanagimachi and Noda, 1970; Moore and Bedford, 1978). In vivo, the acrosome reaction is almost certainly triggered by the zona pellucida, or a cumulus-derived agonist, but the underlying mechanism remains to be unequivocally established (reviewed by Yanagimachi, 1988; Meizel et al., 1990; Saling et al., 1990; Kopf and Gerton, 1991). The acrosome reaction in mammals involves multiple point fusions between the plasma membrane and the adjacent outer acrosomal membrane (Barros et al., 1967; Bedford and Cooper, 1978). Hybrid membrane vesicles are formed, composed of equal proportions of plasma membrane and outer acrosomal membrane (Russell et al., 1979). The equatorial segment of the acrosome does not participate in the acrosome reaction (Barros et al., 1967; Bedford and Cooper, 1978) and the small segment of persisting plasma membrane overlying it is

\footnotetext{
*Permanent address: Department of Zoology, Biology Faculty, General Soedirman University, Purwokerto 53122, Indonesia.

†Present address: Laboratory of Radiobiology and Environmental Health, University of California San Francisco, California, USA.

\$Reprint requests.

Received 5 October 1992
}

the site of sperm-oocyte membrane fusion (Bedford et al., 1979; Moore and Bedford, 1983).

The acrosome reaction has not been described for marsupial spermatozoa. The marsupial acrosome is unusually stable and remains intact in the presence of high concentrations of divalent cations, calcium ionophore A23187 and cyclic nucleotides (Mate and Rodger, 1991). All of these treatments can induce spermatozoa of placental mammals to undergo an acrosome reaction, although their effectiveness varies between species (Santos-Sacchi and Gordon, 1980; Shams-Borhan and Harrison, 1981; Fraser, 1984; Stock and Fraser, 1989; DeJonge et al., 1991). The marsupial acrosome also remains intact after freezethawing in the absence of cryoprotectant, and exposure to the detergent Triton X-100 at concentrations that disturb the sperm plasma membrane (Sistina et al., 1993a). Acrosomal damage, in the presence of cryoprotectants, is a common occurrence when the spermatozoa of placental mammals are frozen (reviewed by Watson, 1979), and detergent treatment has been used to induce an acrosome reaction (Yanagimachi, 1975; Wooding, 1975; Trejo and Mujica, 1990).

Marsupial spermatozoa are essentially similar to mammalian spermatozoa in character but differ in several features due to the $90^{\circ}$ difference in the plane of nuclear flattening during spermiogenesis (marsupials - dorso-ventral, placentals - lateral; reviewed by Rodger, 1991). The marsupial acrosome lies on the dorsal surface of the sperm head and does not form a cap over 
the leading edge. In the species examined here, the acrosome is a compact 'button' adjacent to the leading edge of the sperm head. However, in other Australian marsupial species, and the American opossums, it extends over a large portion of the dorsal surface of the sperm head (e.g. Harding et al., 1979, 1984; Temple-Smith, 1987). There is no evidence that the marsupial acrosome has a morphologically distinct equatorial segment (Harding et al., 1976; Temple-Smith and Bedford, 1976). However, it is unwise to ignore the possibility of functional subregions since the acrosome reaction has yet to be described for any marsupial spermatozoa.

The present study began by examining the possibility that, although calcium ionophore does not induce marsupial spermatozoa to undergo an acrosome reaction, this might be achieved if the ionophore treatment was accompanied by an appropriate diacylglycerol. This followed on from work (Roldan and Harrison, 1989, 1990a; Harrison and Roldan, 1990; Harrison et al., 1990) which demonstrated a role for the phosphoinositide pathway in the regulation of ionophore-induced acrosome reaction in the spermatozoa of placental mammals.

\section{Materials and Methods}

\section{Animals and semen}

Adult, common brushtail possums (Trichosurus vulpecula) were captured in the Newcastle area and housed as described earlier (Rodger and Mate, 1988) in a $14 \mathrm{~h}$ light: $10 \mathrm{~h}$ dark cycle. Tammar wallabies (Macropus engenii) were from the colony of the CSIRO Division of Wildlife and Ecology in Canberra and were housed in grassed yards as described by Mate and Rodger (1991). Animal experimentation was approved by the University of Newcastle Animal Care and Ethics Committee, and the use of protected animals licensed by the New South Wales National Parks and Wildlife Service.

Semen was collected by electroejaculation (Mate and Rodger, 1991) from a pool of four possums and seven wallabies. At least seven days separated successive electroejaculations from any one animal. Spermatozoa were scored for percentage motility $(0-100 \%)$ and progressive motility (0-5) (Rodger $e$ al. 1991). Ejaculates exhibiting poor sperm motility (\% motile $<90 \%$; progressive motility $<4$ ) were discarded. In general, individual sperm samples were used for each experimental replicate, although pooled semen was used on occasions when sperm numbers were low. During the experimental period, possum ejaculates had a sperm concentration of $1.5 \pm$ $0.4 \times 10^{7}$ spermatozoa $\mathrm{ml}^{-1}$ (mean $\pm \mathrm{SEM}$ ) and volume of $3.8 \pm 0.3 \mathrm{ml}(n=26)$. Possum spermatozoa were washed by the 'swim-up' method as described by Mate and Rodger (1991)

Wallaby semen was collected and washed by the 'swim-up' method without dilution. Briefly, 10-20 ml of wallaby semen was collected into a graduated $50 \mathrm{ml}$ centrifuge tube. A small sample of semen was examined immediately for the presence of spermatozoa. The remainder of the semen was left at room temperature for approximately $30 \mathrm{~min}$ to coagulate. Twenty to thirty millilitres of Eagle's minimum essential medium (EMEM) containing $100 \mathrm{iu}$ penicillin $\mathrm{ml}^{-1}$ and $0.01 \mathrm{mg}$ streptomycin $\mathrm{ml}^{-1}$ at $\mathrm{pH} 7.4$ (Cytosystems Sydney) was placed over the coagulated semen and incubated at $35^{\circ} \mathrm{C}$ for $30 \mathrm{~min}$ to allow spermatozoa to 'swim-up'. Sperm concentration in the 'swimup' was $1.3 \pm 0.4 \times 10^{6}$ wallaby sperm $\mathrm{ml}^{-1}$ (mean $\pm \mathrm{SEM}$ ) in $29.0 \pm 2.9 \mathrm{ml}$ of $\operatorname{EMEM}(n=17)$.

\section{Assessment of acrosomal status by light microscopy}

Paraformaldehyde-fixed spermatozoa were attached to microscope slides by centrifugation ('Cytospin', Shandon Inc., Pittsburgh) at $210 \mathrm{~g}$ for $3 \mathrm{~min}$ (Mate and Rodger, 1991) and then stained by the Bryan's technique (Bryan, 1970). Briefly, spermatozoa were fixed for $10 \mathrm{~min}$ by adding $0.1-0.5 \mathrm{ml}$ sperm suspension (depending on sperm concentration) to $2 \mathrm{ml} 2 \%$ paraformaldehyde in PBS ( $\mathrm{pH}$ 7.4). Acrosomes were visualized by staining for 4 min in Bryan's stain (0.1\% eosin Y, Sigma No. E4382; 0.1\% fast green FCF, Sigma No. F7258; 0.1\% flavianic acid, Sigma No. F6500 in $1 \%$ acetic acid: all from Sigma, St Louis, MO). Data were obtained from 600 possum or wallaby spermatozoa per treatment (three slides: 200 spermatozoa per slide). Statistical analysis of acrosome and motility data was by Student's $t$ test after square root transformation.

\section{Assessment of acrosomal status by transmission electron microscopy}

Treated spermatozoa were fixed in $2 / 3$ Superfix or Karnovsky's fixative for $10 \mathrm{~min}$ and then centrifuged at $750 \mathrm{~g}$ for $10 \mathrm{~min}$ to form sperm pellets. The fixation and processing of sperm pellets for electron microscopy has been described (Mate and Rodger, 1991). Thin sections were cut using an Ultracut $\mathrm{E}$ (Reichert-Jung, Vienna) ultramicrotome and stained in uranyl acetate $(10 \mathrm{~min})$, lead citrate $(10 \mathrm{~min})$ and then examined in a Jeol 100-CX electron microscope.

\section{Diacylglycerol treatments}

The diacylglycerols (1,2-dioctanoyl-sn glycerol $\left(\mathrm{DiC}_{8}\right) ; 1,2$ dioleoyl-sn-glycerol (DOG); and 1-oleoyl-2-acetyl-sn-glycerol $(\mathrm{OAG})$ ) tested were obtained from the Sigma. Where possible diacylglycerols were transported frozen to minimize breakdown. Stock solutions of diacylglycerol were made up in dimethylsulfoxide (DMSO) at $10 \mathrm{mmol}^{-1}$ and stored frozen in individual aliquots (containers wrapped in aluminium foil) until required. Calcium ionophore (A23187) was dissolved in DMSO at $1 \mathrm{mg} \mathrm{ml} \mathrm{m}^{-1}$ and stored frozen until required. The reagents were made up at twice the final concentration to be tested in EMEM. Each test solution was mixed 1:1 with the washed sperm suspension and incubated at $35^{\circ} \mathrm{C}$ in $5 \% \mathrm{CO}_{2}$ in air. Control spermatozoa were treated with EMEM containing DMSO at the same concentration as experimental treatments.

\section{Results}

\section{Acrosomal status of marsupial spermatozoa in the presence of diacylglycerols plus calcium ionophore}

Acrosomal loss was evident when possum and wallaby spermatozoa were incubated in the presence of calcium ionphore plus $\mathrm{DiC}_{8}$ or $\mathrm{OAG}$ (Fig. 1). $\mathrm{DiC}_{8}$ was the more potent agent and 

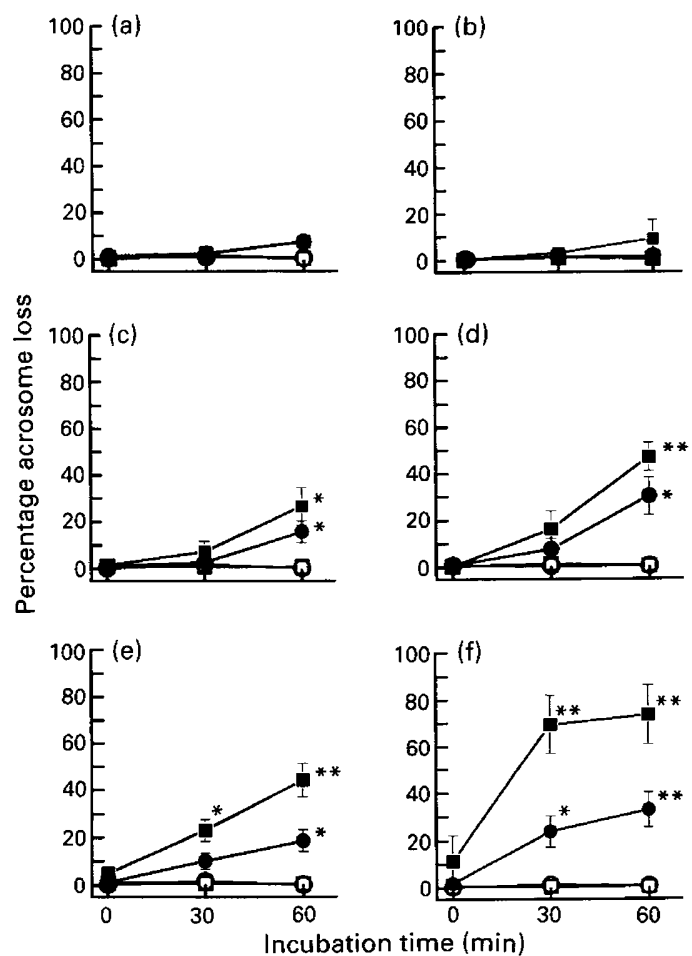

Fig. 1. The percentage of spermatozoa undergoing acrosome loss, as assessed by Bryan's staining, of ( $a, c$ and e) common brushtail possums (Trichosurus vulpecula) and (b, $\mathrm{d}$ and $\mathrm{f}$ ) tammar wallabies (Macropus engenii) after incubation for 30 or $60 \mathrm{~min}$. The incubation medium contained $10 \mu \mathrm{mol}$ calcium ionophore (A23187) $1^{-1}$ plus $50 \mu \mathrm{mol} \mathrm{l}^{-1}(\mathrm{O})$ or $100 \mu \mathrm{mol} \mathrm{l}^{-1}(\boldsymbol{\square})$ of the diacylglycerols: $(\mathrm{a}, \mathrm{b})$ DOG (1,2-dioleoyl-sn-glycerol); (c, d) OAG (1-oleoyl-2-acetyl-snglycerol) and $(\mathrm{e}, \mathrm{f}) \mathrm{DiC}_{8}$ (1,2-dioctanoyl-sn-glycerol). (O) DMSO controls. Significantly different from control: ${ }^{*} P<0.01,{ }^{* *} P<0.001$.

wallaby spermatozoa were the more sensitive. Acrosomal loss did not occur when spermatozoa were incubated with DOG plus ionophore (Fig. 1).

Ten and thirty per cent acrosomal loss was seen after incubation of possum spermatozoa for $30 \mathrm{~min}$ with $50 \mu \mathrm{mol} \mathrm{l}^{-1}$ $(P=0.05)$ and $100 \mu \mathrm{mol} \mathrm{l}^{-1}(P<0.01) \mathrm{DiC}_{8^{\prime}}$ respectively. After $60 \mathrm{~min}$ incubation, acrosomal loss increased to $20 \%$ $(P<0.01)$ and $45 \%(P<0.001)$, respectively. Possum spermatozoa incubated with $50 \mu \mathrm{mol} O A G \mathrm{I}^{-1}$ plus ionophore showed no acrosomal loss after incubation for $30 \mathrm{~min}$, but after 60 min exhibited similar acrosomal loss (15\% and $25 \%)$ to that found with $\mathrm{DiC}_{8}$ plus ionophore after $30 \mathrm{~min}(P<0.01)$. Twenty-five per cent $(P<0.01)$ and $70 \%(P<0.001)$ of wallaby spermatozoa had lost their acrosome after $30 \mathrm{~min}$ incubation with 50 or $100 \mu \mathrm{mol} \mathrm{DiC}_{8} \mathrm{I}^{-1}$, respectively, and there was little change after incubation for a further $30 \mathrm{~min}$. Wallaby spermatozoa incubated with OAG plus ionophore showed no significant acrosomal loss after $30 \mathrm{~min}$, but significant $(30$ to $40 \%)$ acrosomal loss was found after $60 \mathrm{~min}\left(50 \mu \mathrm{mol} \mathrm{l}^{-1}\right.$, $P=0.01 ; 100 \mu \mathrm{mol} \mathrm{l}^{-1} P<0.001$ ).

\section{Acrosomal status after incubation with diacylglycerols alone}

When possum and wallaby spermatozoa were treated with $100 \mu \mathrm{mol}^{-1} \mathrm{DOG}, \mathrm{OAG}$ and $\mathrm{DiC}_{8}$ without calcium ionophore
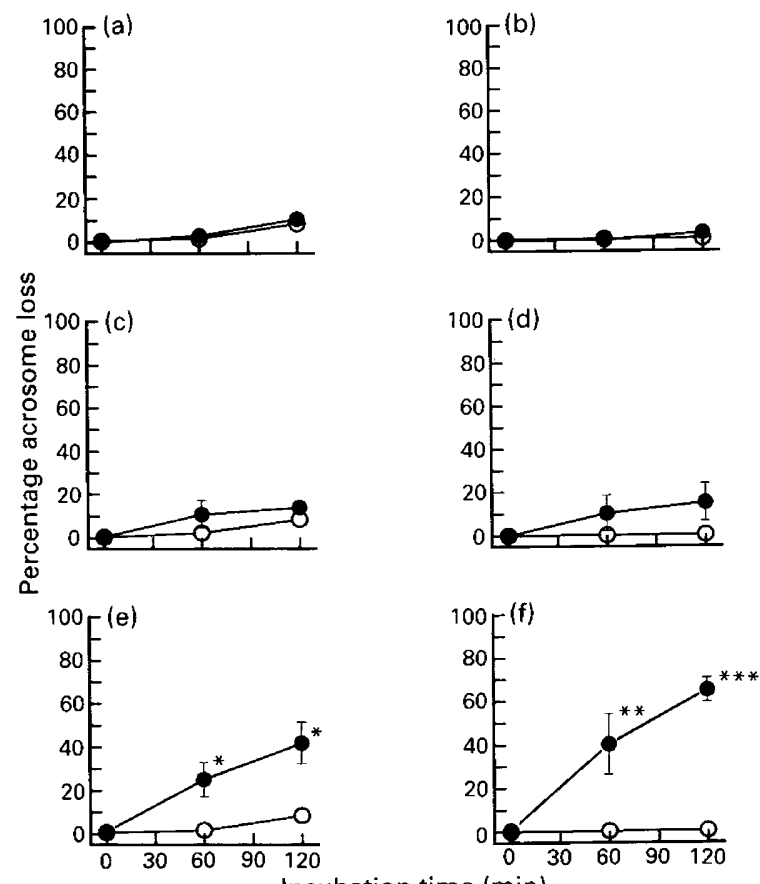

Fig. 2. The percentage of spermatozoa undergoing acrosome loss, as assessed by Bryan's staining, of ( $a, c$ and e) common brushtail possums (Trichosurus vulpecula) and (b, $\mathrm{d}$ and $\mathrm{f}$ ) tammar wallabies (Macropus engenii) after incubation for 60 or $120 \mathrm{~min}$. The incubation medium contained $100 \mu \mathrm{mol} 1^{-1}$ (O) of the diacylglycerols: $(a, b)$ DOG (1,2-dioleoyl-sn-glycerol); (c, d) OAG (1-oleoyl-2-acetyl-snglycerol) and (e, f) $\mathrm{DiC}_{8}$ (1,2-dioctanoyl-sn-glycerol). (O) DMSO controls. Significantly different from control: ${ }^{*} P<0.05$, ${ }^{* *} P<0.01$, ${ }^{* * *} P<0.001$.

(Fig. 2), an essentially similar pattern of acrosomal loss was seen to that found when $10 \mu \mathrm{mol} \mathrm{l}^{-1}$ ionophore was present (Fig. 1). However, similar acrosomal loss was only achieved with $\mathrm{DiC}_{8}$ ( $40 \%$ possum; $70 \%$ wallaby) after a longer period of incubation (120 min).

Twenty-five per cent of possum spermatozoa had lost their acrosome after incubation for $60 \mathrm{~min}$ with $\mathrm{DiC}_{8}(P<0.05)$ and $40 \%$ after $120 \mathrm{~min}(P<0.05)$. Forty per cent of wallaby spermatozoa had lost their acrosome after $60 \mathrm{~min}(P<0.01)$ and $65 \%$ after $120 \mathrm{~min}(P<0.001)$. DOG and OAG did not induce significant acrosomal loss in the absence of ionophore.

Sperm motility in the presence of the diacylglycerols plus calcium ionophore

Spermatozoa treated with each of the three diacylglycerols and calcium ionophore were essentially immotile when examined after incubation for $30 \mathrm{~min}$ (data not shown), although only $100 \mu \mathrm{mol} \mathrm{DiC}_{8} 1^{-1}$ had induced statistically significant acrosomal loss by that time (Fig. 1). In a further experiment, the effect of ionophore on motility loss was examined (Fig. 3). There was no significant loss of motility when wallaby spermatozoa were incubated for $28 \mathrm{~min}$ in the presence of $100 \mu \mathrm{mol}$ $\mathrm{DiC}_{8} \mathrm{l}^{-1}$ and $1 \mu \mathrm{mol}$ calcium ionophore $\mathrm{l}^{-1}$. However, the motility of possum and wallaby spermatozoa had declined significantly after 7 and $14 \mathrm{~min}$, respectively, of incubation with $\mathrm{DiC}_{8}$ plus 5 or $10 \mu \mathrm{mol} 1^{-1}$ ionophore. 


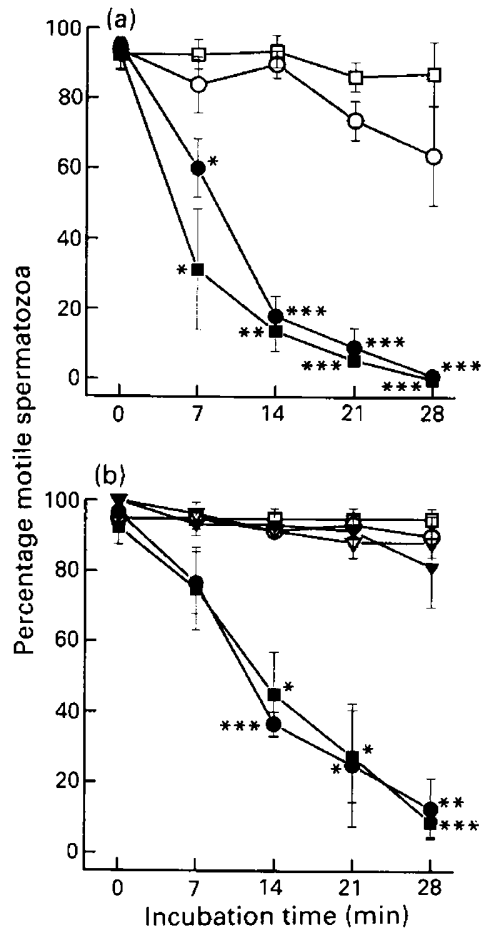

Fig. 3. The percentage of spermatozoa from (a) common brushtail possums (Trichosurus vulpecula) and (b) tammar wallabies (Macropus engenii) that were motile after incubation for $7,14,21$ or $28 \mathrm{~min}$ with $100 \mu \mathrm{mol} \mathrm{I}^{-1}$ of the diacylglycerol $\mathrm{DiC}_{8}$ (1,2-dioctanoyl-sn-glycerol) plus $1 \mu \mathrm{mol} \mathrm{I}^{-1}$ ( $\boldsymbol{\nabla}$; wallabies only), $5 \mu \mathrm{mol} \mathrm{l}^{-1}(\mathbf{)})$ or $10 \mu \mathrm{mol} \mathrm{l} \mathrm{l}^{-1}$ (ם) calcium ionophore (A23187). (O, []) DMSO controls. Significantly different from controls: ${ }^{*} P<0.05,{ }^{* *} P<0.01$ or ${ }^{* * *} P<0.001$

Acrosomal loss was correlated with a decline in motility (Fig. 4). Possum spermatozoa incubated with $100 \mu \mathrm{mol} \mathrm{DiC}_{8} \mathrm{l}^{-1}$ plus $5 \mu \mathrm{mol}$ calcium ionophore $\mathrm{I}^{-1}$ exhibited statistically significant acrosomal loss and a decline in percentage motility after $7 \mathrm{~min}$. Regression of percentage acrosome loss against percentage motility for possum data (Fig. 4) had a correlation coefficient of 0.99 . Wallaby spermatozoa incubated with $100 \mu \mathrm{mol}$ $\mathrm{DiC}_{8} 1^{-1}$ plus $5 \mu \mathrm{mol}$ ionophore $\mathrm{l}^{-1}$ exhibited the same pattern; however, significant acrosomal loss was seen after $7 \mathrm{~min}$ $(P<0.001)$, while a significant decline in motility required $14 \mathrm{~min}$ of incubation. Regression of percentage acrosome loss against percentage motility for wallaby data (Fig. 4) had a correlation coefficient of 0.83 .

\section{Motility of spermatozoa incubated with diacylglycerols alone}

The motility of possum spermatozoa treated with $100 \mu \mathrm{mol}$ $\mathrm{DiC}_{8} \mathrm{l}^{-1}$ differed from controls after $120 \mathrm{~min}$ of incubation $(P<0.05$; Fig. 5). Although motility of possum spermatozoa declined to about $40 \%$ after incubation for $120 \mathrm{~min}$, the motility of spermatozoa treated with $100 \mu \mathrm{mol}$ DOG $~^{-1}$ or $100 \mu \mathrm{mol}$ $\mathrm{OAG}^{-1}$ did not differ from DMSO controls. Incubation of wallaby spermatozoa in the presence of $100 \mu \mathrm{mol} \mathrm{DiC}_{8} \mathrm{l}^{-1}$ led to significant loss of motility by $60 \mathrm{~min}(P<0.01)$ and essentially all spermatozoa were immotile after $120 \mathrm{~min}$ $(P<0.01)$. DMSO-treated control wallaby spermatozoa

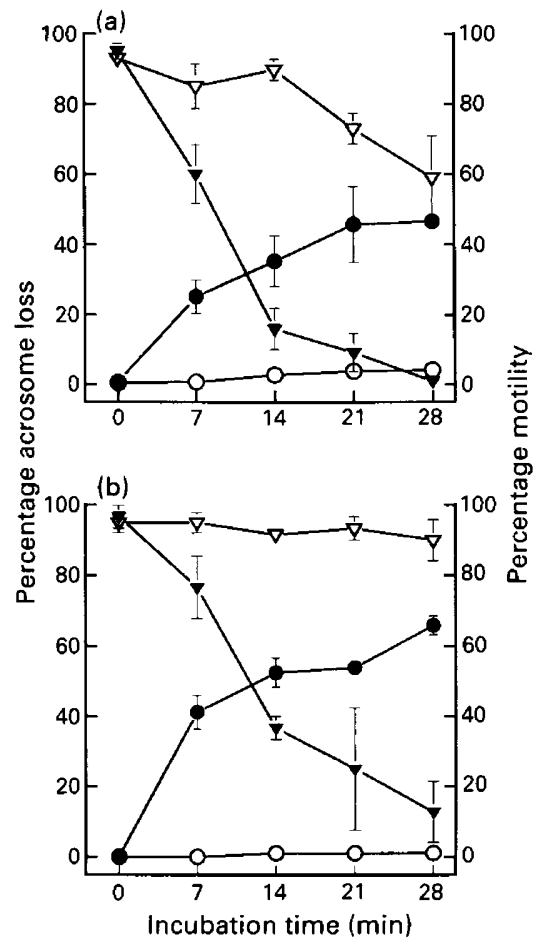

Fig. 4. Percentage acrosome loss $(\boldsymbol{O})$ and percentage motility $(\boldsymbol{\nabla})$ of spermatozoa from (a) common brushtail possums (Trichosurus vulpecula) and (b) tammar wallabies (Macropus eugenii) after incubation for $7,14,21$ or $28 \mathrm{~min}$ with $100 \mu \mathrm{mol} \mathrm{l}^{-1}$ of the diacylglycerol $\mathrm{DiC}_{8}$ (1,2-dioctanoyl-sn-glycerol) plus $5 \mu \mathrm{mol}$ calcium ionophore $\mathrm{I}^{-1}$ (A23187). $(\mathrm{O}, \nabla)$ are DMSO controls. Possum acrosome loss and sperm motility are significantly different from controls after $7 \mathrm{~min}$ $(P<0.001)$. Wallaby acrosome loss is significantly different from control after $7 \mathrm{~min}(P<0.001)$. Wallaby sperm motility is significantly different from controls after $14 \mathrm{~min}$ (14 min $P<0.001 ; 21 \mathrm{~min}$ $P<0.05 ; 28 \mathrm{~min} P<0.01$ ).

showed little loss of motility after incubation for $120 \mathrm{~min}$ and the motility of spermatozoa treated with $100 \mu \mathrm{mol}^{\mathrm{DOG}} \mathrm{l}^{-1}$

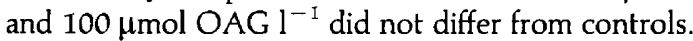

\section{Ultrastructure of $\mathrm{DiC}_{8}$-induced acrosomal loss}

The ultrastructure of the sperm head and its overlying plasma membrane appeared normal in DMSO-treated control spermatozoa (Fig. 6a). The surface of the acrosome of spermatozoa incubated for $5 \mathrm{~min}$ in $100 \mu \mathrm{mol} \mathrm{DiC}_{8} 1^{-1}$ was covered by many membrane vesicles (Fig. 6b). The morphology of the vesicles, and the more osmophilic character of the membrane component adjacent to the acrosome (Fig. $6 \mathrm{~b}$ insert), suggested that they were a hybrid of plasma membrane and outer acrosomal membrane produced by multiple point fusions. Membrane vesiculation was limited to the acrosomal region; the plasma membrane over the remainder of the surface of most spermatozoa remained intact (Fig. 7). After 10 or 30 min incubation in $100 \mu \mathrm{mol} \mathrm{DiC}_{8} \mathrm{l}^{-1}$, the acrosomal matrices were either highly vesiculated, or the acrosome contents and overlying plasma membrane were lost. The inner acrosomal membrane and the plasma membrane over the non-acrosomal surface remained intact in 


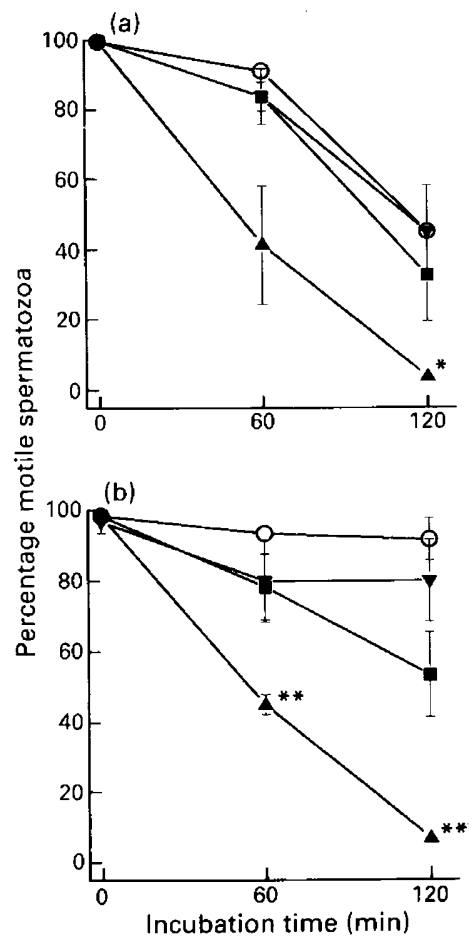

Fig. 5. The percentage of spermatozoa of (a) common brushtail possums (Trichosurus vulpecula) and (b) tammar wallabies (Macropus eugenii) that were motile after incubation for 60 or $120 \mathrm{~min}$ with $100 \mu \mathrm{mol} \mathrm{I}^{-1}$ of the diacylglycerols: ( $\left.\nabla\right)$ DOG (1,2-dioleoyl-snglycerol); ( $\mathbf{\square})$ OAG (1-oleoyl-2-acetyl-sn-glycerol) and $(\boldsymbol{\Delta}) \mathrm{DiC}_{8}(1,2-$ Dioctanoyl-sn-glycerol). (O) DMSO controls. Significantly different from controls: ${ }^{*} P<0.05,{ }^{* *} P<0.01$.

most spermatozoa that had lost the acrosome. Incubation with $\mathrm{DiC}_{8}$ and $10 \mu \mathrm{mol}$ calcium ionophore $\mathrm{A} 23187 \mathrm{I}^{-1}$ did not alter this pattern of ultrastructural change, but did result in more generalized cellular disruption than when spermatozoa were incubated with $\mathrm{DiC}_{8}$ alone.

\section{Discussion}

The diacylglycerol $\mathrm{DiC}_{8}$ (1,2-dioctanoyl-sn-glycerol) was found to induce acrosomal loss in $70 \%$ of wallaby spermatozoa and $40 \%$ of possum spermatozoa after incubation for $120 \mathrm{~min}$. If $10 \mu \mathrm{mol}$ calcium ionophore $\mathrm{I}^{-1}$ were present, the time required to reach these end points was reduced to 30 and $60 \mathrm{~min}$, respectively. The diacylglycerol OAG (1-oleoyl-2-acetyl-snglycerol) produced some acrosomal loss when $10 \mu \mathrm{mol}$ calcium ionophore $\mathrm{I}^{-1}$ was present, but not when ionophore was absent. The other diacylglycerol tested, DOG (1,2-dioleoyl-snglycerol), did not induce significant acrosomal loss in possum or wallaby spermatozoa, alone, or in combination with ionophore.

The finding that the three diacylglycerols tested in this study differed in their ability to induce acrosomal loss is consistent with previous studies. Diacylglycerol analogues differ in their ability to induce biological responses because derivatives with short, unsaturated fatty acid side chains compete for the binding sites of protein kinase $\mathrm{C}$ more effectively than those with satu-
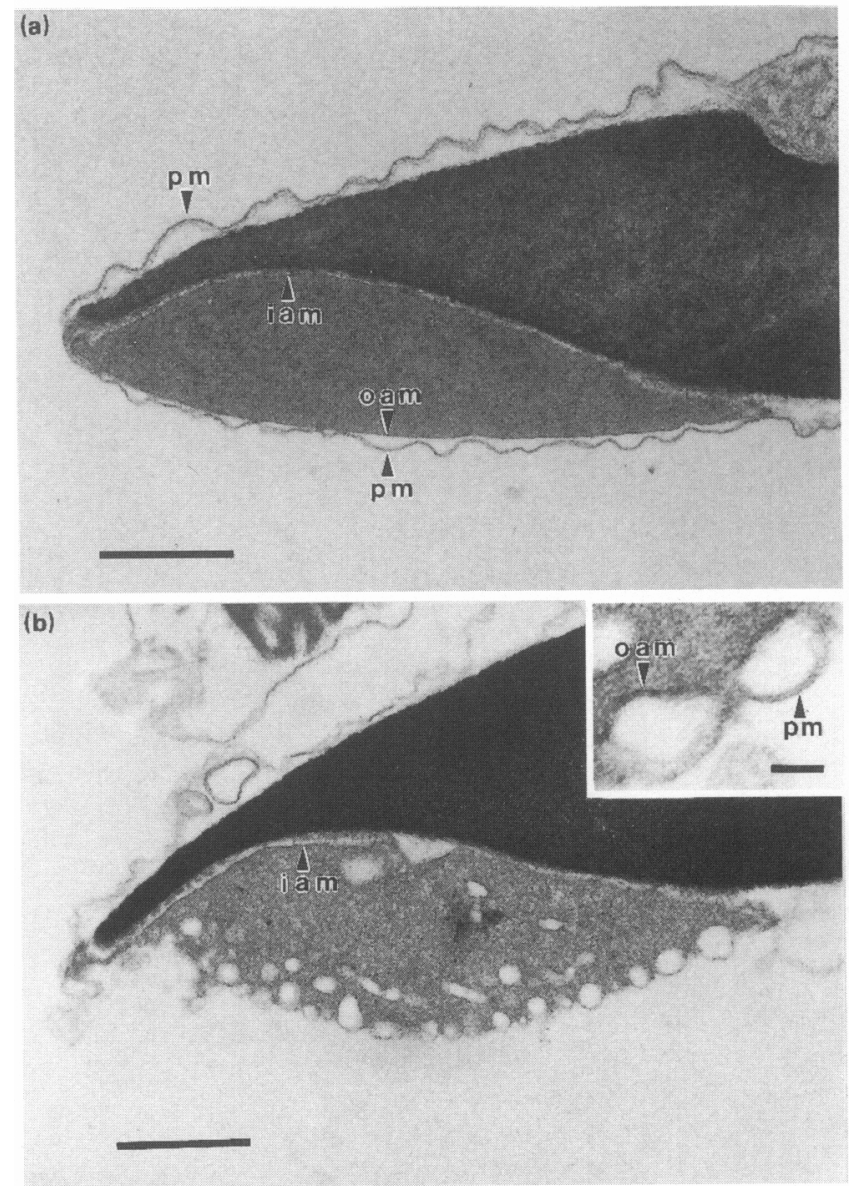

Fig. 6. Electron micrographs of the anterior head of spermatozoa from tammar wallabies (Macropus engenii) spermatozoa. pm: plasma membrane; oam: outer acrosomal membrane; iam: inner acrosomal membrane. (a) DMSO control incubated for $5 \mathrm{~min}$. The intact plasma membrane is loosely applied to the outer acrosomal membrane on the smooth convex surface of the acrosome. (b) Spermatozoa incubated with $100 \mu \mathrm{mol} \mathrm{DiC}_{8} \mathrm{I}^{-1}$ (1,2-dioctanoyl-sn-glycerol) for $5 \mathrm{~min}$. The plasma membrane and outer acrosomal membranes have undergone multiple point fusions. There is also evidence of the early stages of vesiculation of the acrosomal matrix. (b Insert) Detail of plasma membrane outer acrosomal membrane point fusion. Note the difference in electron density of the two membranes. Scale bars: $0.5 \mu \mathrm{m}$ (insert bar $0.07 \mu \mathrm{m}$ ).

rated fatty acid side chains (Ebeling et al., 1985; Leach and Blumberg, 1989). However, the concentrations of $\mathrm{DiC}_{8}$ required to induce the acrosome reaction in marsupial spermatozoa (50$100 \mu \mathrm{mol} \mathrm{l}^{-1}$ ) were high compared with the range of concentrations used in other studies $\left(0.1-100 \mu \mathrm{mol} \mathrm{l}^{-1}\right.$; Kramer et al., 1987; Lee et al., 1987; Burch, 1988; Roldan and Harrison, 1990b), suggesting a mode of action other than enzyme activation.

Preincubation of wallaby spermatozoa with suboptimal concentrations of $\mathrm{DiC}_{8}\left(10 \mu \mathrm{mol} l^{-1}\right.$ or $\left.20 \mu \mathrm{mol} \mathrm{l}^{-1}\right)$ before addition of $\mathrm{A} 23187\left(10 \mu \mathrm{mol} \mathrm{l}^{-1}\right)$ led to little acrosomal loss (10.5 $\pm 3 \%$; 60 min each treatment). This was not statistically different from spermatozoa incubated in 10 or $20 \mu \mathrm{mol} \mathrm{DiC}_{8}$ $\mathrm{I}^{-1}$ alone (data not shown). No significant acrosomal loss was detected when possum or wallaby spermatozoa were treated 


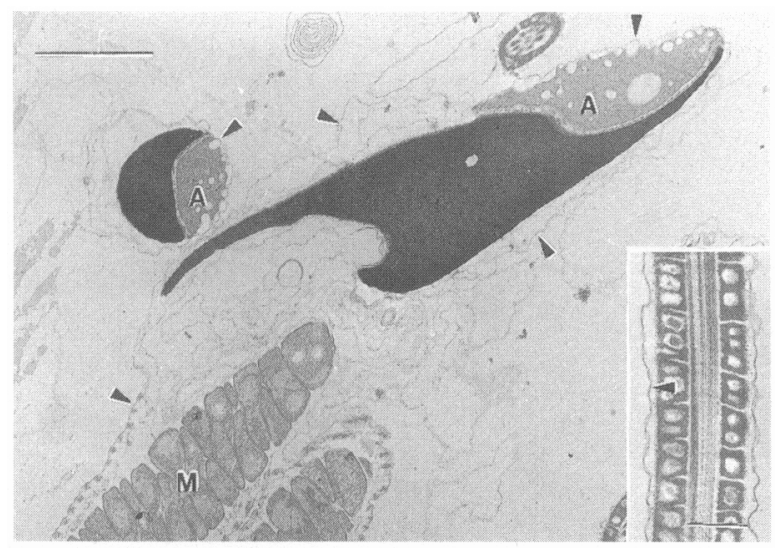

Fig. 7. Electron micrographs of spermatozoa from tammar wallabies (Macropus engenii) after incubation in $100 \mu \mathrm{mol} \mathrm{DiC}_{\mathrm{g}} \mathrm{I}^{-1}(1,2-$ dioctanoyl-sn-glycerol) for $5 \mathrm{~min}$. The main figure is of sections of sperm heads and mid-pieces (scale bar: $1.0 \mu \mathrm{m}$ ). The insert is a higher power detail of a region of the principal piece of a sperm tail from the same treatment group (scale bar: $0.25 \mu \mathrm{m}$ ). A: acrosome; $\mathrm{M}$ : mitochondria of the mid-piece; arrowheads: plasma membrane. In the sperm heads the plasma membrane overlying the acrosome is undergoing vesiculation due to multiple point fusions with the underlying outer acrosomal membrane and there is some vesiculation of the acrosomal matrices. The non-acrosomal plasma membrane of the sperm heads and the plasma membrane of the mid-pieces and principal piece are intact without any evidence of vesiculation or fusion.

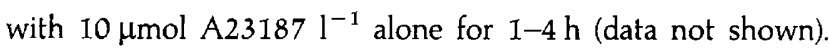
These findings agree with earlier work from this laboratory which found that acrosomal loss by possum and wallaby spermatozoa was not induced by A23187 (Mate and Rodger, 1991).

Sperm motility was rapidly compromised in the presence of calcium ionophore (5 or $10 \mu \mathrm{mol} \mathrm{l}^{-1}$ ) after incubation for $28 \mathrm{~min}$, and it is unlikely that many spermatozoa were viable after incubation for $30 \mathrm{~min}$. However, despite the deleterious effects of ionophore treatment, a significant number of wallaby spermatozoa had lost their acrosome (7 min; Fig. $4 \mathrm{~b}$ ) before a significant decline in motility was detected (14 min; Fig. $4 \mathrm{~b}$ ). The statistically significant acrosomal loss, found for possum and wallaby spermatozoa incubated with $O A G$ and ionophore for $60 \mathrm{~min}$, is likely to reflect major cellular disruption rather than a diacylglycerol/ionophore-induced acrosome reaction. Of the three diacylglycerols tested, only $\mathrm{DiC}_{8}$ led to significant loss of motility when used without A23187. However, this occurred only after relatively long incubation periods (wallaby: $60 \mathrm{~min}$; possum: $120 \mathrm{~min}$; data not shown).

The ultrastructure of the acrosomal loss induced by $\mathrm{DiC}_{8^{\prime}}$ point fusions of the outer acrosomal membrane and overlying plasma membrane, was essentially identical to the physiological acrosome reaction described for a broad range of placental mammals (reviewed by Yanagimachi, 1988). The ultrastructure of the vesiculation observed in wallaby spermatozoa treated with $\mathrm{DiC}_{8}$ suggested that, as in placental mammals, they were formed of hybrids of plasma membrane and outer acrosomal membrane. Onset of point fusion between the outer acrosomal membrane and its overlying plasma membrane was detected after incubation for $5 \mathrm{~min}$ with $\mathrm{DiC}_{8}$. Significant loss of motility was not detected until $30 \mathrm{~min}$ exposure to the diacylglycerol.
After 30 min incubation, most spermatozoa had lost all traces of the outer acrosomal membrane and acrosomal matrix, although the inner acrosomal membrane and adjacent nuclear envelope were intact (a detailed ultrastructural examination of the $\mathrm{DiC}_{8}$ induced acrosomal loss will be the subject of a subsequent paper). The non-acrosomal plasma membrane of spermatozoa undergoing acrosomal loss remained intact.

The manner of acrosomal loss induced by $\mathrm{DiC}_{8}$ treatment was quite different from that seen when possum and wallaby spermatozoa were treated with the detergent Triton X-100 (Sistina et al., 1993a) or lysophosphatidylcholine (Sistina et al., 1993b). In these cases, the first structure disturbed was the plasma membrane and it was affected over the entire surface of the spermatozoa. Disruption of the acrosomal matrix and membranes followed, but there was no evidence of plasma membrane-outer acrosomal membrane fusion. These studies and our earlier work on marsupial spermatozoa have demonstrated that the acrosome and its membrane are unusually stable compared with the acrosomes of the spermatozoa of placental mammals (Mate and Rodger, 1991; Rodger et al., 1991). We have not observed outer acrosomal membrane instability/ fusogenicity in any of our previous work.

The mechanism of acrosomal loss induced by $\mathrm{DiC}_{8}$ in marsupial spermatozoa remains unclear. The effective concentrations of $\mathrm{DiC}_{8}$ were much higher than those used in studies of the acrosome reaction in placental mammals and in other phosphoinositide pathway systems (e.g. Roldan and Harrison, 1990b). Yet the morphology of acrosomal loss induced mimicked that seen in the physiological acrosome reaction of placental mammals and not that seen when the marsupial acrosome is lost after disruptive treatments (Sistina et al., 1993a, b). A companion paper (Mate and Rodger, 1993) has examined the mechanism of the $\mathrm{DiC}_{8}$-induced acrosomal loss and concluded that it was most likely that the diacylglycerol acted as a direct membrane fusogen.

The study was undertaken with the financial support of a grant to J. C. Rodger from the Australian Research Council. Y. Sistina was the recipient of a Fellowship from the International Development Program of Australian Universities and Colleges and K. E. Mate was supported by an Australian Postgraduate Research Award. We are indebted to E. Roldan for his encouragement to pursue the diacylglycerols and for advice on their use and handling.

\section{References}

Barros C, Bedford JM, Franklin LE and Austin CR (1967) Membrane vesiculation as a feature of the mammalian acrosome reaction Journal of Cell Biology 34 CI-C5

Bedford JM and Cooper GW (1978) Membrane fusion events in the fertilization of vertebrate eggs. In Membrane Fusion, pp 65-125 Eds G Poste and GL Nicolson. Elsevier/North Holland, New York

Bedford JM, Moore HDM and Franklin LE (1979) Significance of the equatorial segment of the acrosome of the spermatozoon in eutherian mammals Experimental Cell Research 119 119-126

Bryan JHD (1970) An eosin-fast green-naphthol yellow mixture for differential staining of cytologic components in mammalian spermatozoa Stain Technology 45 231-236

Burch RM (1988) Diacylglycerol stimulates phospholipase A2 from Swiss 3T3 fibroblasts FEBS Letters 234 283-286

De Jonge CJ, Han HL, Lowrie H, Mack SR and Zaneveld LJD (1991) Modulation of the human sperm acrosome reaction by effectors of the adenylate 
cyclase/cyclic AMP second-messenger pathway Journal of Experimental Zoology 258 113-125

Ebeling JG, Vandenbark GR, Kuhn LJ, Ganong BR, Bell RM and Niedel JE (1985) Diacylglycerols mimic phorbol diester induction of leukemic cell differentiation Proceedings National Academy of Science USA 82 815-819

Fraser LR (1984) Mechanisms controlling mammalian fertilization. In Oxford Reviews of Reproductive Biology, Vol. 6 pp 174-225 Ed. CA Finn. Clarendon Press, Oxford

Harding HR, Carrick FN and Shorey CD (1976) Ultrastructural changes in spermatozoa of the brush-tailed possum, Trichosurus vulpecula (Marsupialia), during epididymal transit, part II: the acrosome Cell and Tissue Research 171 61-73

Harding HR, Carrick FN and Shorey CD (1979) Special features of sperm structure and function in marsupials. In The Spermatozoon, pp 289-303 Eds DW Fawcett and JM Bedford. Urban and Schwarzenberg, Baltimore

Harding HR, Carrick FN and Shorey CD (1984) Sperm ultrastructure and development in the honey possum, Tarsipes rostratus. In Possums and Gliders, PP 451-461 Eds AP Smith and ID Hume. Surrey Beatty and Sons and the Australian Mammal Society, Sydney

Harrison RAP and Roldan ERS (1990) Phosphoinositides and their products in the mammalian sperm acrosome reaction Journal of Reproduction and Fertility Supplement 42 51-67

Harrison RAP, Roldan ERS, Lander DJ and Irvine RF (1990) Ram spermatozoa produce inositol 1,4,5-trisphosphate but not inositol 1,3,4,5-tetrakisphosphate during the $\mathrm{Ca}^{2+}$ /ionophore-induced acrosome reaction Cellular Signalling 2 $277-284$

Kopf GS and Gerton GL (1991) The mammalian sperm acrosome and the acrosome reaction. In Elements of Mammalian Fertilization, Vol. 1 Basic Concepts, pp 153-203 Ed. PM Wassarman. CRC Press, Boca Raton, Florida

Kramer RM, Checani GC and Deykin D (1987) Stimulation of $\mathrm{Ca}^{2+}$-activated human platelet phospholipase $A_{2}$ by diacylglycerol Biochemical Journal 248 779-783

Leach KL and Blumberg PM (1989) Tumour promoters, their receptors and their actions. In Inositol Lipids in Cell Signalling, pp 179-205 Eds RH Michell, AH Drummond and CP Downes. Academic Press, London

Lee MA, Kopf GS and Storey BT (1987) Effects of phorbol esters and a diacylglycerol on the mouse sperm acrosome reaction induced by the zona pellucida Biology of Reproduction 36 617-627

Mate KE and Rodger JC (1991) Stability of the acrosome of the brush-tailed possum (Trichosurus vulpecula) and tammar wallaby (Macropus eugenii) in vitro and after exposure to conditions and agents known to cause capacitation or acrosome reaction of eutherian spermatozoa joumal of Reproduction and Fertility $9141-48$

Mate KE and Rodger JC (1993) Role of diacylglycerols and calcium in the marsupial acrosome reaction journal of Reproduction and Fertility 99 367-373

Meizel S, Pillai MC, Diaz-Perez E and Thomas P (1990) Initiation of the human sperm acrosome reaction by components of human follicular fluid and cumulus secretions including steroids. In Fertilization in Mammals pp 205-222 Eds BD Bavister, J Cummins and ERS Roldan. Serono Symposia, Norwell, Massachusetts

Moore HDM and Bedford JM (1978) An in vivo analysis of factors influencing the fertilization of hamster eggs Biology of Reproduction 19 879-885

Moore HDM and Bedford JM (1983) The interaction of mammalian gametes in the female. In Mechanism and Control of Animal Fertilization, pp 453-497 Ed. JF Hartmann. Academic Press, New York

Rodger JC (1991) Fertilization of marsupials. In A Comparative Overview of Mammalian Fertilization, pp 117-135 Eds BS Dunbar and MG O'Rand. Plenum Press, New York

Rodger JC and Mate KE (1988) A PMSG/GnRH method for the superovulation of the monovulatory brush-tailed possum (Trichosurus vulpecula) Journal of Reproduction and Fertility 83 885-891
Rodger JC, Cousins SJ and Mate KE (1991) A simple glycerol-based freezing protocol for the semen of a marsupial Trichosurus vulpecula, the common brushtail possum Reproduction Fertility and Development 3 119-125

Roldan ERS and Harrison RAP (1989) Polyphosphoinositide breakdown and subsequent exocytosis in the $\mathrm{Ca}^{2+}$ /ionophore-induced acrosome reaction of mammalian spermatozoa Biochemical Journal 259 397-406

Roldan ERS and Harrison RAP (1990a) Molecular mechanisms leading to exocytosis during the sperm acrosome reaction. In Fertilization in Mammals, pp 179-196 Eds BD Bavister, J Cummins and ERS Roldan. Serono Symposia, Norwell, Massachusetts

Roldan ERS and Harrison RAP (1990b) Diacylglycerol and phosphatidate production and the exocytosis of the sperm acrosome Biochemical and Biophysical Research Communications 172 8-15

Russell L, Peterson R and Freund M (1979) Direct evidence for formation of hybrid vesicles by fusion of plasma and outer acrosomal membranes during the acrosome reaction in boar spermatozoa journal of Experimental Zoology $20841-56$

Saling PM, Bunch DO, Le Guen P and Leyton L (1990) ZP3-induced acrosomal exocytosis: a new model for triggering. In Fertilization in Mammals, pp 239-252 Eds BD Bavister, J Cummins and ERS Roldan. Serono Symposia, Norwell, Massachusetts

Santos-Sacchi J and Gordon M (1980) Induction of the acrosome reaction in guinea pig spermatozoa by cGMP analogues Journal of Cell Biology $\mathbf{8 5}$ 798-803

Shams-Borhan G and Harrison RAP (1981) Production, characterization, and use of ionophore-induced, calcium-dependent acrosome reaction in ram spermatozoa Gamete Research 4 407-432

Sistina Y, Lin M, Mate KE, Robinson ES \& Rodger JC (1993a) The unique stability of the marsupial sperm acrosomal membranes examined by unprotected freeze-thawing and treatment with the detergent Triton X-100 Reproduction Fertility and Development 5 1-14

Sistina Y, Lin M and Rodger JC (1993b) Lysophosphatidylcholine disrupts the acrosome of the tammar wallaby (Macropus eugenii) Molecular Reproduction and Development 35 277-284

Stock CE and Fraser LR (1989) Divalent cations, capacitation and the acrosome reaction in human spermatozoa Journal of Reproduction and Fertility $\mathbf{8 7}$ 463-478

Temple-Smith PD (1987) Sperm structure and marsupial phylogeny. In Possums and Opossums: Studies in Evolution, pp 171-193 Ed. M Archer. Surrey Beatty \& Sons and Royal Zoological Society of NSW, Sydney

Temple-Smith PD \& Bedford JM (1976) The features of sperm maturation in the epididymis of a marsupial, the brushtailed possum Trichosurus vulpecula American Journal of Anatomy 147 471-499

Trejo $\mathbf{R}$ and Mujica A (1990) Changes in calmodulin compartmentalization throughout capacitation and acrosome reaction in guinea pig spermatozoa Molecular Reproduction and Development 26 366-376

Watson PF (1979) The preservation of semen in mammals. In Oxford Reviews of Reproductive Biology, Vol. I pp 283-350 Ed. CA Finn. Clarendon Press, Oxford

Wooding FBP (1975) Studies on the mechanism of hyamine-induced acrosome reaction in ejaculated bovine spermatozoa Journal of Reproduction and Fertility 44 185-192

Yanagimachi R (1975) Acceleration of the acrosome reaction and activation of guinea pig spermatozoa by detergents and other reagents Biology of Reproduction 13 519-526

Yanagimachi R (1988) Mammalian fertilization. In The Physiology of Reproduction, Vol. 1 pp 135-185 Eds E Knobil and JD Neill. Raven Press, New York

Yanagimachi R and Noda YD (1970) Physiological changes in the post-nuclear cap region of mammalian spermatozoa: a necessary preliminary to the membrane fusion between sperm and egg cells journal of Ultrastructure Research $\mathbf{3 1}$ 486-493 